\title{
RESPONSE OF YBCO/PBCO/YBCO RAMP TYPE JOSEPHSON JUNCTIONS TO NEAR MM WAVE IRRADIATION.
}

\author{
D. Terpstra, G.J. Gerritsma and H. Rogalla.
}

Department of Applied Physics, University of Twente, P.O. Box 217, 7500 AE Enschede, The Netherlands.

R. Gupta and Q. Hu.

Department of Electrical Engineering and Computer Science and Research Laboratory of Electronics, Massachusetts Institute of Technology, Cambridge, Massachusetts 02139, USA.

A high $\mathrm{T}_{\mathrm{c}}$ Josephson device for high frequency detection applications is being developed, consisting of an $\mathrm{YBCO} / \mathrm{PBCO} / \mathrm{YBCO}$ ramp type junction and a broad band log-periodic antenna. In this contribution we present the response of such a device to (near) $\mathrm{mm}$ wave irradiation. Shapiro steps have been observed up to very high voltage values - nearly $4 \mathrm{mV}$ at $10 \mathrm{~K}$, at the maximum of the radiation power. The modulation of the step amplitudes shows very good resemblence with the predictions from the Resistively Shunted Junction model.

\section{INTRODUCTION}

High $T_{c}$ Josephson junctions are very attractive for high frequency applications. To make a competitive device, the junctions must meet many requirements $[1,2]$. The first and most important one is a good response to (sub-) millimeter-wave radiation. Shapiro steps should be observable up to voltages of at least several $\mathrm{mV}$ and should modulate with radiation power according to the RSJ model.

We are developing a device for high frequency applications using YBCOIPBCOIYBCO ramp type junctions, placed between the terminals of a logperiodic antenna to improve the coupling to external radiation [1-3]. Design consideratations, the fabrication process of the junctions, and their scaling and Josephson properties have been discussed in detail $[1,2,4]$.

In this contribution we present the response of this type of junctions to near $\mathrm{mm}$ wave radiation.

The work at Twente was supported by the Foundation for Fundamental Research on Matter (FOM) and the Netherlands Technology Foundation (STW). The work at MIT was conducted under the auspices of the Consortium for Superconducting Electronics with full support by DARPA (contract MDA972-90-C-0021).

\section{RESULTS}

The presented measurements were performed on two devices, $\mathrm{A}$ and $\mathrm{B}$. A has the described layout, with $\mathrm{T}_{\mathrm{c}} \approx 80 \mathrm{~K}, \mathrm{I}_{\mathrm{c}} \approx 280 \mu \mathrm{A}$ at $10 \mathrm{~K}$ and a temperature independent $R_{n} \approx 3.5 \Omega$. B was not equipped with an antenna; $\mathrm{T}_{\mathrm{c}} \approx 75 \mathrm{~K}, \mathrm{I}_{\mathrm{c}} \approx 100 \mu \mathrm{A}$ at $7.5 \mathrm{~K}$ and $\mathrm{R}_{\mathrm{n}} \approx 2.5 \Omega$. Both devices were fabricated on YSZ substrates, and had a PBCO barrier thickness of about $10 \mathrm{~nm}$.

Irradiation of $\mathrm{A}$ with a $\mathrm{mm}$ wave signal causes the appearance of many Shapiro steps. Figure 1 shows the current-voltage characteristics (IVc's) at $10 \mathrm{~K}, 35 \mathrm{~K}$ and $65 \mathrm{~K}$. The DC IVc's are shown, together with those for maximum applied $100 \mathrm{GHz}$ power. At $10 \mathrm{~K}$, the $19^{\text {th }}$ step can still be observed at a bias voltage of $4 \mathrm{mV}$. This corresponds to the presence of $\mathrm{AC}$ Josephson oscillations with a frequency of $1.9 \mathrm{THz}$. At higher temperatures the number of observed steps decreases: 13 are present at $35 \mathrm{~K}, 5$ at $65 \mathrm{~K}$. At all temperatures steps are observed up to voltages far above the $I_{c} R_{n}$ product.

Device B, irradiated with a $176 \mathrm{GHz}$ signal, also exhibits Shapiro steps up to high voltages: at $4.2 \mathrm{~K}$, with maxmimum signal power, at least the $6^{\text {th }}$ step is still observable at $2.2 \mathrm{mV}$, which corresponds to $1.05 \mathrm{THz} \mathrm{AC}$ Josephson oscillations [5]. 


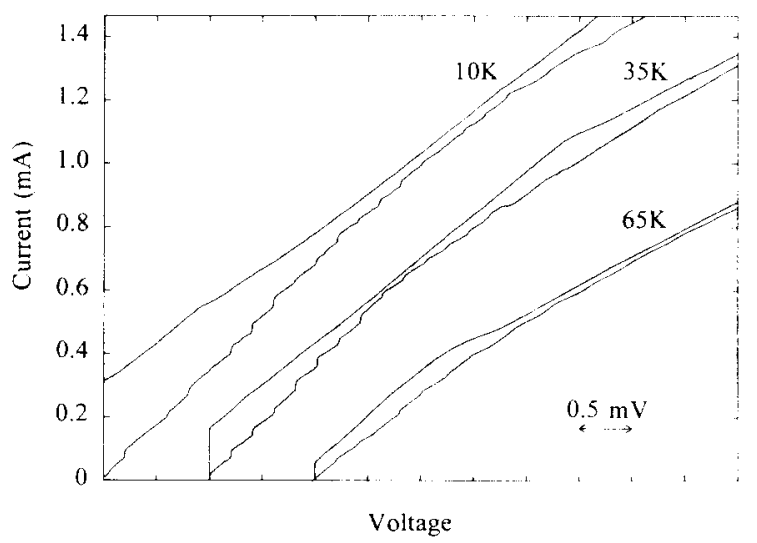

Figure 1. Current voltage characteristics of device $A$ at $10 \mathrm{~K}, 35 \mathrm{~K}$ and $65 \mathrm{~K}$, both $\mathrm{DC}$ and with $100 \mathrm{GHz}$ irradiation at the maximum power level.

Only few studies are present in literature that report high frequency response up to similar voltage values. Those results, however, were obtained with devices of a less controllable type, e.g. [6]

With increasing power the Shapiro steps demonstrate the proper oscillating behavior. Figure 2 shows for device $B$ at $8 \mathrm{~K}$ the normalized amplitudes of $I_{c}$ and the first two Shapiro steps $I_{1,2}$ as a function of $2 \alpha=\mathrm{C}_{\mathrm{RF}} \mathrm{P}_{\mathrm{RF}}{ }^{1 / 5}$, where $\mathrm{P}_{\mathrm{RF}}$ is the applied $176 \mathrm{GHz}$ signal power. The measured data agree very well with a Bessel function behavior $I_{n} \sim J_{n}(2 \alpha)$, which is expected for this high frequency [5]. $\mathrm{C}_{\mathrm{RF}}$ was used as fitting parameter, in such a way that the first zero of $J_{1}(2 \alpha)$ coincides with that of $I_{1}$ [5].

For device A, with $100 \mathrm{GHz}$ irradiation, a similar good agreement was found between the measured amplitudes of $I_{c}$ and $I_{1,2}$ and the predictions of the RSJ model [2] at several temperatures.

\section{CONCLUSIONS}

The presented results show that ramp type YBCO/PBCO/YBCO Josephson junctions have a very high degree of phase locking, up to voltages of at least $4 \mathrm{mV}$, despite the strong reduction of the $I_{c} R_{n}$ product. In addition, the amplitudes of $I_{c}$ and the Shapiro steps are well described by the RSJ model.

All these results indicate that these junctions are
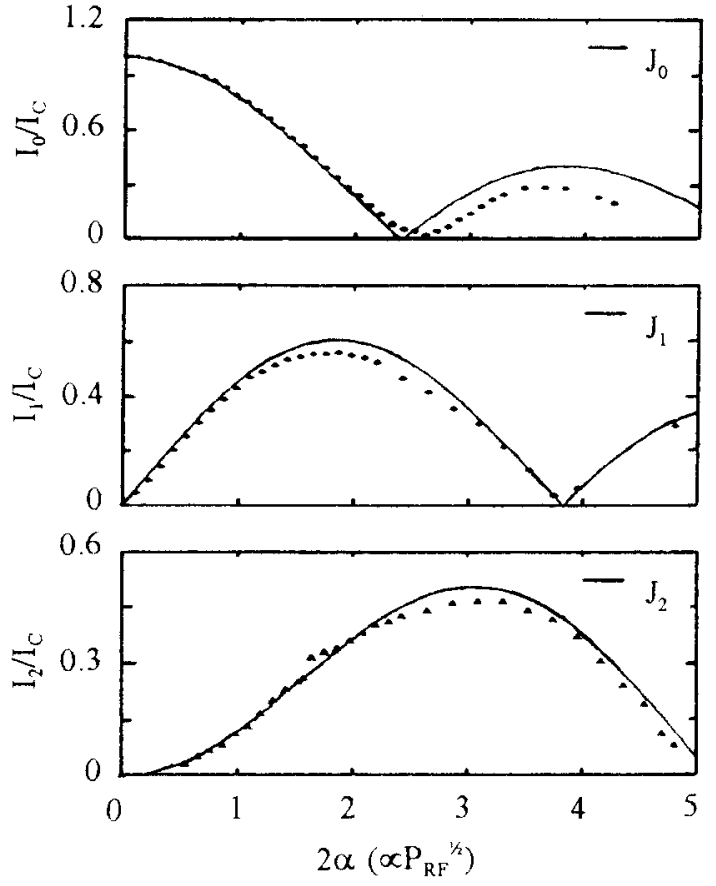

Figure 2. Measured normalized amplitudes of $I_{c}$ and $\mathrm{I}_{1,2}$ of device $\mathrm{B}$ as a function of $2 \alpha \sim \mathrm{P}_{\mathrm{RF}}{ }^{1 / 2}$ at $176 \mathrm{GHz}$ near $8 \mathrm{~K}$. The solid lines are Bessel functions.

very suitable for (sub-) mm-wave applications.

What the limiting frequency for such applications is, and to what extent it is limited by the specific transport properties of the junctions is under investigation.

\section{REFERENCES}

[1] D. Terpstra, A.J.H.M. Rijnders, F.J.G. Roesthuis, D.H.A. Blank, G.J. Gerritsma and H. Rogalla, to be published in J. Alloys and Compounds.

[2] in preparation.

[3] P.H. Siegel, IEEE MTT-S Digest (1986) 649.

[4] J. Gao, Yu.M. Boguslavskij, B.B.G. Klopman, D. Terpstra, R. Wijbrans, G.J. Gerritsma and H. Rogalla, J. Appl. Phys. 72 (1992) 575.

[5] R. Gupta, Q. Hu, D. Terpstra, G.J. Gerritsma and $H$. Rogalla, submitted for publication.

[6] J. Takeya, E. Kawate, C.H. Park and T. Goto, Appl. Phys. Lett. 61 (1992) 2601. 